\title{
Research on Reactive Power Compensation Based on Distribution Network
}

\author{
Ling Wang \\ Benxi Power Supply Branch, State Grid Liaoning Electric Power Supply Co. Ltd \\ Benxi, China \\ bxwl_wl@163.com
}

\begin{abstract}
The power is a essential resource in the life of people. With the continuous expansion of the city and covering an area of the grid more widely, it is difficult to control the growing supply. The stability and the change of the power in the power system have a direct relationship. An effective way to improve the power factor is to take the appropriate means of reactive power compensation. Reactive power compensation mainly refers to the effective use of reactive power compensation devices and equipment. In order to obtain the required reactive power, it reduces the energy consumption of grid operation and promote the power system effectively improve the power factor, and ultimately achieve improved grid voltage quality goals. In power system optimization process, through the application of reactive power compensation modules, it will be more conducive to enhance the voltage quality. Therefore, it is great significance to the study of reactive power compensation for the social development.
\end{abstract}

Keywords- expansion; supply; power compensation; consumption; application

\section{INTRODUCTION}

Transmission grid users in the process, power to be supplied to the electric power load in two ways: active and reactive power. Active means to keep equipment running the required electric power, which is the electrical energy into other forms of energy (mechanical energy, light, heat, etc.) of electric power; without power refers to power electrical equipment inductors, capacitors and other components work electric power required to establish the magnetic field[1]. It is mainly used for the exchange of energy within the electric and magnetic fields of electrical equipment, the establishment and maintenance of the power of the magnetic field in electrical equipment (circuit system). Although no external work, the role is very important. Motor rotating magnetic field needed for the operation, which is by reactive power to establish and maintain, with the rotating magnetic field, in order to make the rotor, thus boosting the mechanical operation. Transformers also require reactive power, generate a magnetic field in order to make the primary coil, the secondary coil induces a voltage. Where there is solenoid electrical equipment operation requires the establishment of a magnetic field, however, the establishment and maintenance of the energy consumed by the magnetic field from the reactive power[2]. No reactive power, the motor does not rotate, the transformer can not run the relay will not operate all devices in the field can not be established; the electrical equipment will not run. Therefore, it is in addition to the power supply system to provide users with real power, but also to provide reactive power.

In the power system, there are many professional equipment, each device there is a certain amount of load, which determines the size of the load changes in the value of power factor. Power factor is the power system is an important beam data, it may reflect the use of low-power electrical equipment, work efficiency, scientific workers who hold the power system, control and stability of the power system has practical significance. Power factor, the higher the efficiency of the power system, the greater the ratio of useful work and total power, so long must continue to improve the supply of power factor value, thereby increasing the economic benefits of the plant at all. So what is an effective means of increasing the power factor is it? According to summarize the experiment and experience, we have found that the use of reactive power compensation method can significantly improve the power factor, most plants will also apply this method to transport electricity[3].

If the generators and high-voltage transmission lines to supply the equipment a lot of reactive power, power factor then becomes very small, active power supply will far failed to meet the needs of the load, while also resulting in decreased quality of power supply, the net loss increases First, an increase in reactive power means that the reactive current increases, so that the total current transmission line increases, the impedance of the transmission line under certain circumstances, result in increased current consumption increases lines and equipment; secondly, if the grid reactive power capacity is inadequate, will cause the load side of the power supply voltage decreases. If the reactive excess capacity will cause the load side of the power supply voltage is too high, affecting the normal production and life of electricity; third, in terms of power generation equipment, reactive current increases, increasing the demagnetization effect on the generator rotor, voltage reduction, if excessive excitation current increases, the rotor windings will exceed the allowable temperature rise[4].

(A) Improve the electricity supply system and load power factor, reduce equipment capacity, reduce power loss; stable receiving end voltage, grid voltage can be maintained within the allowable range, thereby reducing the voltage fluctuations to improve power quality, thus effectively reducing the power loss and power consumption, extend the life of the equipment.

(B) The appropriate locations in the long-distance transmission lines in dynamic reactive power 
compensation device can also improve the stability of the transmission system, improving transmission capacity; In addition, the reactive power compensation to reduce the reactive current transformer, so that the power transformer reduce losses and increase supply capacity; after output of the generator reactive power compensation reactive current reduction, can also increase the active power output of the generator.

(C) In the case of the three-phase unbalanced load, through appropriate reactive power compensation can balance three-phase active and reactive loads.

\section{THE SIGNIFICANCE OF REACTIVE POWER COMPENSATION}

Distribution network is an important part of the power system. On the one hand, the distribution network direct contact with users, and its power supply reliability and power quality directly affects the development of the national economy and people's daily life, it is necessary to ensure the safe operation of the distribution grid. On the other hand, low voltage distribution network, network losses account for a large proportion of the entire power network, thus achieving optimal operation of the distribution network, to improve the economic power enterprises has important significance[5].

Duing to historical reasons, a problem of the construction and operation of transmission and distribution network is a long-standing lack of reactive power compensation capacity and equipped unreasonable, especially lack of reactive power capacity can be adjusted, fast response less reactive power conditioning equipment. In recent years, with the increasing power of nonlinear loads, a large number of polluting large electricity failed to take effective measures to control the harmonic voltage fluctuations, power shock caused by flicker, negative sequence and other electrical pollution, grid reactive power and harmonic pollution impact was a rising trend. On the other hand, the lack of reactive power regulation means making changes in the way the bus voltage with a great run, lead to the grid (in particular, the distribution system) line loss increases, the voltage passing rate. According to statistics, the loss rate in rural areas of China's highpressure part of the more than $20 \%$, which is mainly reflected in the $10 \mathrm{kV}$ distribution network, "August" the end of the national rural network's average net loss of $28 \%$. The effective way to reduce line losses is to improve the reactive power distribution grid, reactive power compensation. Therefore, from the perspective of reducing the net loss in the distribution network for reactive power compensation huge role, has great economic value.

In the distribution network, the majority of users the power factor is low, and without compensation device, coupled with the distribution network is characterized by long lines, distribution points, light load, load multi-use characteristics of squirrel-cage induction motor, resulting in a with low power factor, energy efficiency is not high, poor quality of power supply situation. Therefore, the distribution network for reactive power compensation, not only can reduce the burden on the level of the grid, but also can improve the utilization of distribution transformers, power factor and power users to improve quality, and can effectively reduce the net loss for the user and the electricity sector has enormous significance.

Reactive Power Compensation conduct an effective measure is to develop user-Power Technology. It is the FACTS technology to develop applications in low voltage distribution grid, the purpose is to improve the power quality of the user side. The concept was first user power technology proposed in 1988 by Dr. NGHingorani, which is an application of modern electronic technology, computer technology and control technology, according to user specific requirements and provides power supply to achieve the power quality control technology. Its core is the power electronics, micro-processing and control technology in the distribution system is to enhance a comprehensive economic transient power quality and effective method. Some Chinese scholars called D-FACTS, FACTS technologies that this is an extension of the application in the distribution system, and a lot of research work. Compared with FACTS, its controller principle and structure of the same, just smaller electrical unit value, its purpose is to strengthen the reliability of power supply and distribution system, and improve power quality. Insulated gate bipolar transistors, integrated gate commutated thyristor and other devices based on the three-phase voltage source is a major component of the various $\mathrm{CP}$ controller have faster switching frequency, so it has fast response characteristics, can be the perfect solution to transient power quality problems[6].

\section{REACTIVE POWER COMPENSATION MEANS}

\section{A. Traditional reactive power compensation}

Traditional reactive power compensation equipment synchronous condenser, fixed capacitors, switches, switching shunt reactors, these devices can meet a range of reactive power compensation requirements, but they also exist inherent drawbacks.

Fixed compensation capacitor is used in the highpressure side of the system for centralized compensation, but it has a major way of compensation, a wide range of applications. It has a low cost, low active power loss, no rotating parts, simple installation, easy maintenance, etc., but only compensation fixed capacitor reactive power, can not achieve a smooth continuous automatic adjustment, sometimes with the system harmonics parallel resonance occurs, the short-circuit stability is also poor. In addition, dramatic changes in load, less likely to cause reactive power compensation, compensation can not be dynamic light load reactive overpower compensation and overloading[7].

Synchronous condenser: Until the 1970s, synchronous condenser is the only available power system, fully controllable reactive power compensation device. It is designed to produce reactive power synchronous motor, which is not possible with a mechanical load overexcitation (often running state) or under excitation (less running) synchronous motor running. When the system voltage is low, over-excitation of the reactive power supply and raise the system voltage; under-excitation of 
the high absorption system when excess reactive power in the system and the system voltage to lower voltage. It can be two-way, continuously adjustable; can be adjusted independently by adjusting the reactive excitation size, greater overload capacity. The disadvantage is that its time constant of the field winding greater control response is slow (100 500ms), high investment in equipment, startup, operation and maintenance of complex, even more expensive than the same capacity static compensator.

Saturated Reactor: 1967, the British company GEC made the world's first saturation reactor type static reactive power compensation device. Since then, national manufacturers have launched their own products. Saturated reactor compared with synchronous condenser has a static type of advantage that the response speed, but because of its core needs to saturation magnetization, and thus loss and noise are great and there are some special problems of nonlinear circuits, they can not phase adjusted to compensate for load imbalance, we could not occupy the mainstream static var compensator device. .

\section{B. Static var compensator}

SVC static reactive power compensation device referred to generally refer specifically to the use of thyristor reactive power compensation equipment, is currently the most widely used in the power system, the most sophisticated equipment tied compensation. IEEE wills SVC defined as a parallel-type static var generator or absorber, its output can be adjusted to the exchange of capacitive or inductive current, so as to maintain or control the power system specific parameters[8].

Thyristor power electronic devices such as static control element reactive power compensation device is an advanced high-voltage power grid dynamic power factor compensation device, there are fast response, flexible control, continuously adjustable and so on. With the development of power electronic devices and computer control technology, SVC is moving in the direction of high-voltage and high-capacity development. The current SVC has been widely used in load compensation and compensation modern power transmission line system to improve the stability of the power system and suppress voltage fluctuations applied to shock loads (such as electric arc furnace) flicker suppression and non-electrified railway balance compensation among power companies around the world and is recognized as a mature industry and effective reactive power management tools and apply it.

SVC mainly in the following three types: thyristor controlled reactance, hyristor switched capacitor and thyristor controlled high leakage reactance transformer type static var compensation device. Due to advances in technology, mixed-use of these devices belong to static reactive power compensation device, such as TSC-TCR type mixing device, or thyristor controlled reactor TCR fixed capacitor of the mixing device that TCR-FC, or thyristor controlled reactor TCR and mechanically switched capacitor mixed-use device that TCR-MSC type.

In the current engineering practice, the choice of TCR- FC type static var compensator devices accounted for the majority. Because TCR can be continuous reactive power control, three phase power and improve the ability to better flicker, restricted the ability of over-voltage, reactive power regulation capacity, low power factor, load fluctuations particularly fast reactive power compensation occasion has a high value, and can get better compensation effect. And mixing device FC has better filtering effect, harmonics generated not only can filter out TCR itself, but also the harmonics generated by load fluctuations filtered to reduce the impact on system power quality. By the stepdown transformer (limited to power electronic devices, SVC can not be directly connected to high pressure, high voltage power grid can only access through a step-down transformer) secondary winding is connected to "open star", the midpoint separately, you can make each phase load with the other two phases separate, and thus the positive and negative sequence amplitude can be individually controlled, phase adjustment, can balance the unbalanced load.

\section{Synchronous static var compensator}

Although SVC reactive system can effectively compensate, but due to the commutation element is not drying capacity, making it easier to produce more power harmonic currents, but also for the ability to regulate voltage fluctuations less than ideal. With high-power fullcontrolled thyristor GTO and the IGBT appear as a basis of STATCOM has also become a hot topic. Both are connected in parallel with the grid, from the reactive power support role, but a performance perspective, STATCOM superior to the SVC.

STATCOM and SVC some outstanding compared with the following advantages:

When SVC is by passive devices (inductors, capacitors) to produce reactive power, run outside when SVC voltage adjustment range, the output current proportional to the voltage, that voltage is reduced, the output reactive current (compensation capacity) is also small, which the point is not desirable, but also not as good as a synchronous condenser SVC characteristics (output current and voltage independent of) the most important point. The STATCOM is an active structure, the GTO inverter and DC capacitor constituted by the on-off control of GTO achieve energy exchange between the three-phase, to produce the desired reactive current. When the voltage decreases, STATCOM can still produce a large capacitive current, regardless of the voltage[9].

Good controllability, the voltage amplitude and phase can be adjusted quickly, typically a few milliseconds. Its terminal voltage of the operating conditions and the structure of the external system is not sensitive. Therefore, STATCOM can not only get a better static stability and better performance can be obtained transient stability under large disturbance fault. Due to the small capacity of the capacitor STATCOM in widespread use in the power grid does not produce low-frequency resonance.

SVC is a capacitive reactance and thyristors, and the same STATCOM and other FACTS devices are using high-power electronic devices[10]. The former is the use of traditional materials, prices rise with the passage of time, the latter using a new type of semiconductor material is 
rapidly developing, the price decline over time. In the past few years, the price of GTO to 10 percent per year decline, the recent emergence of IGCT and electron injection enhanced gate transistor (Injection Enhanced Gate Transistor, IEGT) GTO cheaper in price than several tens of percent, while the performance and reliability also better than the GTO, but with the expansion of the scale compared to the use of traditional materials prices have more space.

With the gradual development of computers and communications technologies and the gradual maturation of advanced technology has been found involved in these industries, which naturally includes the use of grid operation. The application of advanced science and technology brought us convenient but also gives us a lot of need to rethink and solve problems. The main problem is the lack of information related to data analysis grid operation. The grid operation, the huge system, which includes data on the digital simulation of the data in the system all the actual test data collected by means of, for example, also each management aspects of management information systems, and a variety of software on the data. As engineering and technical personnel, should be to integrate all of the data coupled with detailed analysis and processing, and finally extract the relevant information to ensure the security and stability of the entire power system operation. But in fact not the case, all the information is part of the information of all the engineering and technical personnel can only get from the data, which are more valuable information buckling modes and the law of development of the power system as well as internal relations and other important but not available, However, more research is precisely the information value.

\section{CONCLUSION}

In summary, the rational use of reactive power compensation to increase the power factor can maintain the stability of power system operation can reduce unnecessary waste of power, can increase economic power plant. Power factor and the size of the power system has a large rigid, invisible power factor by many factors, such as inductive devices reactive power loss, the power supply voltage and rated voltage does not match and so on. Therefore, the staff should pay particular attention to the plant reactive power compensation method, by saving electricity plant expenses, improve equipment utilization, reduce energy systems and other methods to increase the power factor, thereby maximize the protection of the safety of the power system reliable, and promote the stable development of the plant.

\section{ACKNOWLEDGMENT}

The authors gratefully acknowledge the contribution of co-workers and reviewers' comments.

\section{REFERENCES}

[1] P.Y.Feng and Y.H.Li "Power factor and reactive power compensation system of the plant," Ene. Env,vol.12,pp.21-25, July 2010

[2] YY.Jing "Energy efficient method of plant power supply system,' Elec.Inform, vol.12,pp.12-16, May 2010

[3] H.X.Ren and X.Fu, "Factory supply power compensation," Management \& Technology, vol. 8,pp.12-14, April 2010

[4] X.L.Dai, "Reactive power compensation in distribution network applications," Grid technology,vol.6,pp.11-14, July 2009

[5] Y.Z.Han and G.G.Yan "Power and FACTS and DFACTS information technology," Pow.Sys.Auto, vol.24,pp.1-7, July 2000

[6] Z.H.Wang and F.Shen, "FACTS technology and its application and development in China," Pow.Sys.Auto,vol.23,pp.1-5,August 2000

[7] Group I S S C. Static Var Compensator Models For Power Flow And Dynamic Performance Simulation. IEEE Trans.Power Syst., 1994, 9(1): 22-24

[8] J.H.Luo, "STATCOM dynamic characteristics for wind farm reactive power compensation analysis," Pow.Sys.Auto .vol.14,pp.24-27, May 2010

[9] M.Zeng and G.Tian, "Grid substation operation research and analysis techniques ," Power Technology, vol.18 ,pp.22-27, April 2010

[10] Q.Wang, "Substation electrical equipment installation and commissioning, operation and maintenance," Power Technology. vol. 9,pp. 21-24, April 2011 